\title{
DE UN USO GRÁFICO DE CATÓN (QUINT. I 7, 23 y IX 4, 39)
}

According to Quintilianus (I 7, 23; IX 4, 39) Cato used to write dicae or faciae instead of dicam or faciam. However, some scholars have casted doubts on this testimony. This paper tries to show that Cato's dicae and faciae were congruent in the context of Latin grammar and should therefore not be emended.

Nos informa en dos pasajes Quintiliano de una práctica de Catón (I 7, 23 Winterbottom): Quid? non Cato Censorius 'dicam' et 'faciam' 'dicae' et 'faciae' scripsit, eundemque in ceteris quae similiter cadunt modum tenuit? Quod et ex ueteribus eius libris manifestum est et a Messala in libro de s littera positum; y (IX 4, 39-40): Inde 'belligerare', 'pos meridiem' et illa Censori Catonis 'dicae' 'faciae'que, $m$ littera in e mollita. Quae in ueteribus libris reperta mutare imperiti solent, et dum librariorum insectari uolunt inscientiam, suam confitentur. Atqui eadem illa littera, quotiens ultima est et uocalem uerbi sequentis ita contigit ut in eam transire possit, etiam si scribitur, tamen parum exprimitur, ut 'multum ille' et 'quantum erat', adeo ut paene cuiusdam nouae litterae sonum reddat. Neque enim eximitur sed obscuratur, et tantum in hoc aliqua inter duas uocales uelut nota est, ne ipsae coeant.

Desde antiguo estos pasajes han sido diversamente enmendados en relación al grafo utilizado por Catón, aunque dicae, faciae están bien testimoniados y son lecciones de los dos más antiguos manuscritos ( $\mathrm{Co}$ dex Bernensis y Codex Bambergensis, ambos del siglo x) para I 7, 23 '. Conocemos de cuatro propuestas básicas ${ }^{2}$.

O. Ribbeck ${ }^{3}$ propone dice, facie y en IX 4 leería et illa Catonis dice

' Cf. C. H. Moore, "Cato's Final m: a Note to Quint. Inst. Or. I 7, 23: IX 4, 39", AJPh 19, 1898, p. 312.

${ }_{2}$ No hemos tenido acceso a la propuesta de Th. Bergk (Beiträgen zur lateinischen Grammatik, 1879, I, p. 153): dicee, faciee, que resulta insatisfactoria.

3 "Nochmals diee», $R h M 25,1870$, p. 429. 
facieque, A littera in E mollita... atqui eadem illa $M$ littera, pero los manuscritos presentan simplemente illa littera, y la secuencia illa $m$ resultaría injustificada, ya que no se habría hecho antes mención explicita de $m$.

C. H. Moore ${ }^{4}$ cree que la grafia catoniana seria una $\langle\mathrm{M}\rangle$ volcada, algo así como $\langle\Sigma\rangle$, escrita tras la vocal (DICA $\Sigma$ ) o sobre ella, lo que ciertamente habría provocado la confusión del signo con $\langle\mathrm{E}\rangle$, y cree además que $m$ littera in e mollita sería una glosa tempranamente introducida en el texto. M. Niedermann ${ }^{5}$ agregó que el signo aparecería en el libro de Mesala de $s$ littera a causa de la afinidad entre $\langle\Sigma\rangle$ y el sigma $\langle\Sigma\rangle$. Pero, de acuerdo a la dirección dextrorsum de la escritura latina, esperariamos una $\langle\mathrm{M}\rangle$ volcada hacia el otro lado, esto es, $\langle\mathbf{3}>$, que evidentemente ya no presentaría tal afinidad ni con $<\mathrm{E}>$ ni con $\langle\Sigma\rangle$.

Para A. Klotz ${ }^{6}$ estaríamos aquí ante el grafema verriano del que nos habla Velio Longo (VII 80 GLK): ...sicut Verrius Flaccus, ut ubicumque prima uox $m$ littera finiretur, sequens a uocali inciperet, $m$ non tota, sed pars illius prior tantum scribiretur. El signo seria, pues, aproximadamente $\langle\uparrow>$, pero esto difícilmente habría propiciado la confusión con $<E>$ de los manuscritos, aparte de que Verrio, escritor de un opúsculo de obscuris Catonis (Gell., XVII 6), es al menos un siglo posterior al Censor.

R. O. Fink ${ }^{7}$ ha propuesto $<F>(=[\mathrm{f}])$. Cree Fink que la inexistencia de $\langle\mathrm{F}\rangle$ final y el parecido con $\langle\mathrm{E}\rangle$ habrian propiciado el cambio en los manuscritos. Pero aun menos verosímil nos parece la pronunciación efectiva [f] que Fink cree resultado de la relajación de $[-\mathrm{m}]$. No hay ningún otro dato en latín que apunte a esto ni parece bien motivado un paso [m $>\mathrm{f}]$.

Nos parece, sin embargo, válida la lectura dicae, faciae y que hay que entender a Quintiliano uerbatim, o, mejor, litteratim. Éste no indica que Catón representase con $<-\mathrm{e}>$ toda $<-\mathrm{m}>$, como a veces parece entenderse, sino sólo en dicam, faciam... eundemque in ceteris quae similiter cadunt modum.

Un grupo $^{8}$-ae era el único prosódicamente homologable al de -am

4 AJPh 19, 1898, p. 313, con aprobación de R. G. Kent, The Sounds of Latin, N. York $1966(=1932)$, p. 38.

5 Précis de phonétique historique du latin, París $1953_{2}$, p. 102; idem E. Faria, Fonética histórica do Latim, Río de Janeiro $1957_{2}$, p. 97.

" "Über einen Sprachgebrauch Catos", $R h M$ 80, 1931, p. 142.

7 "A Long Vowel Before Final M in Latin", AJPh 90, 1969, p. 451.

${ }^{8}$ Grupo al menos en el sentido grafemático, < ae >, aunque para nosotros además en el fonemático, /ae/. 
(o - $m$ con otra vocal), ya que su valencia métrica normal era, ante vocal, nula (Verg. Aen. I 25 cäusae ìrärūm como Lucr. I 58 mätěrĭem èt gènĭtäliă) y larga ante consonante (Lucr. I 58 quāe nōs mätěrĭem como Verg. Aen. I 25 ìrārùm sãeuiquĕ). Catón habría utilizado tal práctica sólo para primeras personas verbales en -am, nunca habría escrito un acusativo < rosae > por obvios motivos, ni por obvios motivos utilizado $<$ ae $>$ para formas terminadas en -em, -im, - um. Simplemente el latín no disponía de ningún otro diptongo final, pues ni $a u$ ni $o e$ aparecen en tal posición ".

¿Se refiere Quintiliano a todas las formas personales en -am (laudabam, audiam, diceham, dixeram...)? Creemos que más bien sólo a futuros imperfectos de indicativo y presentes de subjuntivo de la tercera conjugación (facère, dicěre), ya que de otro modo no se entendería la monótona insistencia en los ejemplos y el eundem... modum. Significativa es la confrontación con un pasaje de Festo: Recipie apud Catonem, pro recipiam, ut alia eiusmodi complura (286 b 21 Müller). $\mathrm{K} l o t z^{10} \mathrm{re}$ cuerda que recipiae sería aquí la lección manuscrita. Catonianos parecen asimismo por diversos motivos "los otros ejemplos: attinge pro attingam (26, 13 Müll.), dice pro dicam (72, 6 Müll.), ostende, ostendam (201 a 23 Müll.), donde siempre tenemos formas de la III conjugación. Como es sabido, esta conjugación contiene, entre otras, la particularidad de presentar una forma dicam común para presente (dicas) y futuro (dices). Ello ya fue sentido como una anomalia por los antiguos y hubo, junto a involuntarias confusiones, intentos de regularización (Cic. Leg. III 20, 49 faciem $)^{12}$. Lo que Quintiliano ya no nos dice es si Catón escribía < dicae > como una grafia discriminatoria ${ }^{13}$ sólo para el futuro o bien, como una especie de compromiso que subrayaba la identidad en el significante, para ambos tiempos ${ }^{14}$.

${ }^{9}$ No entran en consideración diptongos excepcionales o marginales; de éstos además de $a u(h a u)$, también $e u, o i$ y $u i$ podian ser finales (neu, quoi, hui).

${ }^{10} R h M 80,1931$, p. 141.

"Cf. Klotz, ibidem.

12 Cf. A. Ernout, Morphologie historique du latin, París $1974_{3}$, p. 160.

${ }_{13}$ Al modo, por ej., como Lucilio (cf. Quint. I 7, 15; Vel. Long. VII 56 GLK; Char. I $79 G L K$; Ter. Scaur. VII 18 s. y aun 32 s. $G L K$; = Lucil. IX 353-63 Krenkel) propondrá distinguir $<\mathrm{i}>$ de $<\mathrm{ei}>$ para notación de $i$ (vg. gen. pueri - nom. puerei), ídem Nigidio (ap. Gell. XIII 26, 4) y -ai gen. frente a -ae dat. (uso quizá también luciliano: IX 365 Krenk.; para nom. pl. -ae y gen. sing. -äi v. Quint. I 7. 18-19, también dat. -ai; Vel. Long. VII 57 s. GLK).

14 Es claro que el testimonio de Gelio no basta para atribuir una pronunciación monoptonga de ae por parte de Catón (XVI 12, 7-8): M. Varro... faenus autem dictum ait a fetu et quasi a fetura quadam pecuniae parientis atque increscentis. Idcirco et $M$. Catonem et ceteros aetatis eius feneratorem sine a littera pronuntiasse tradit, sicuti fetus ipse et fecunditas appellata. 
El porqué el expediente catoniano emergía en un libro de s littera, tampoco parece difícil de explicar: [-s] era la única consonante que compartía debilitamiento con [-m]. Mesala podía así relacionar ambos fenómenos. El texto en su contexto (IX 4, 38 Wint.): Quae fuit causa et Seruio < Sulpicio >, ut dixi, subtrahendae s litterae quotiens ultima esset aliaque consonante susciperetur, quod reprehendit Luranius, Messala defendit. Nam neque Lucilium putat uti eadem ultima, cum dicit 'Aeserninus fuit' et 'dignus locoque', et Cicero in Oratore ${ }^{15}$ plures antiquorum tradit sic locutos. Inde 'belligerare'... En defensa de su tesis Mesala habría mencionado el antecedente catoniano.

Mollire no presenta acepción gramatical especializada ${ }^{16}$, pero la metáfora quedaria ya suficientemente explicada por la diferencia cualitativa entre consonante y vocal.

En cualquier caso, el uso $<$ dicae $>$ del Censorio estaba bien justificado*.

\section{Ballester}

${ }^{15} \mathrm{Cf}$. Cic. Or. 161: Ita non erat ea offensio in uersibus, quam nunc fugiunt poetae noui. Sic enim loquebamur 'qui est omnibu' princeps' non 'omnibus princeps', et 'uita illa dignu' locoque' non 'dignus'.

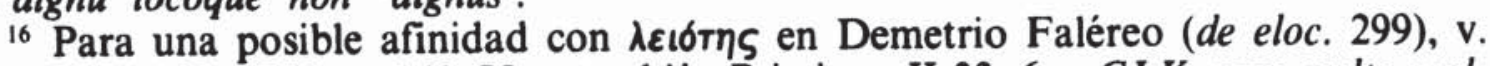
Klotz, $R h M 80,1931$, p. 140 . Y v. también Prisciano II 33, 6 s. GLK: nam multo molliorem et uolubiliorem sonum habet $\psi$ quam ps uel bs.

* Este trabajo se ha beneficiado de una ayuda para el proyecto PB-04091 del programa sectorial de promoción general del conocimiento patrocinado por la Secretaría de Estado de Universidad e Investigaciones. 